\title{
OPTICAL SPECTROSCOPY OF INGAN EPILAYERS IN THE LOW INDIUM COMPOSITION REGIME
}

\author{
M. H. Crawford, J. Han, M. A. Banas, S. M. Myers, G. A. Petersen and J. J. Figiel
}

Sandia National Laboratories, Albuquerque, NM 87185

\begin{abstract}
Photoluminescence (PL) spectroscopy was carried out on a series of Si-doped bulk InGaN films in the low indium (In) composition regime. Room temperature PL showed a factor of 25 increase in integrated intensity as the In composition was increased from 0 to 0.07 . Temperature dependent PL data was fit to an Arrhenius equation to reveal an increasing activation energy for thermal quenching of the PL intensity as the In composition is increased. Time resolved PL measurements revealed that only the sample with highest In $(x=0.07)$ showed a strong spectral variation in decay time across the $T=4 \mathrm{~K}$ PL resonance, indicative of recombination from localized states at low temperatures. The decay times at room temperature were non-radiatively dominated for all films, and the room temperature (non-radiative) decay times increased with increasing In, from 50-230 psec for $\mathrm{x}=0-0.07$. Our data demonstrate that non-radiative recombination is less effective with increasing In composition.
\end{abstract}

\section{INTRODUCTION}

While a great deal of progress has been made in the development of InGaNbased light emitters, the role played by indium (In) in contributing to the optical efficiency is still quite controversial. A number of groups have proposed that the inhomogeneity of In incorporation results in carrier localization at In-rich regions and that this localization leads to enhanced optical efficiency [1-3]. Support of this hypothesis is found in cathodoluminescence experiments that demonstrate a variation of the PL emission energy on the microscale, suggesting that In composition variations on the order of several percent are possible [4]. Further insight is suggested by the timeresolved spectroscopy experiments of InGaN quantum well structures performed by Narukawa, et. al. [5], which suggest that the density of non-radiative centers and possibly the non-radiative recombination mechanism itself is altered when In is included in the growth. A similar result was obtained by Kumano, et. al. [6] who suggest that increased optical efficiency is due to reduced non-radiative recombination centers with In incorporation. These two phenomena, namely localization and reduced non-radiative recombination, may be linked if dislocations act as non-radiative centers [7,8], and if the presence of dislocations also affects the inhomogeneity of In incorporation [8,9], thereby affecting carrier localization. In contrast to these theories, other experiments [10] suggest that the majority of optical spectroscopy data on InGaN quantum wells can be explained entirely by piezoelectric field effects. Thus, it is clear that a strong consensus has not emerged as to whether localization is indeed the primary mechanism by which InGaN alloys and quantum wells achieve high optical efficiencies.

In this paper, we explore these issues through an examination of the effect of In composition on the optical properties of InGaN alloys. Our studies include temperature-

F99W11.41 
dependent and time-resolved photoluminescence spectroscopy measurements on a number of MOVPE growth $\operatorname{In}_{\mathrm{x}} \mathrm{Ga}_{1-\mathrm{x}} \mathrm{N}$ epilayers in the low In composition regime $(\mathrm{x}<$ 0.10). This composition regime was chosen to examine whether a clear trend in optical efficiency, temperature dependent quenching of PL intensity and PL decay time can be found with the addition of just small amounts of In. Our work has also intentionally focused on relatively thick $(0.2 \mu \mathrm{m})$ and doped bulk InGaN epilayers so that the role of piezoelectric field effects would be minimized [11]. This work is therefore distinct from the majority of the previously reported work that has focused on InGaN quantum wells with relatively high $(\mathrm{x} \geq 0.2)$ In composition.

\section{EXPERIMENT}

The MOVPE growth is carried out in a vertical rotating-disc reactor. All of the InGaN epilayers were grown on sapphire substrates and $1 \mu \mathrm{m}$ thick $\mathrm{GaN}$ epilayers $\left(1050^{\circ} \mathrm{C}\right)$ using a standard two-step nucleation procedure with low-temperature $\mathrm{GaN}$ grown at $550{ }^{\circ} \mathrm{C}$. The growth temperature of the InGaN and GaN samples was $800{ }^{\circ} \mathrm{C}$, with the exception of the $\mathrm{InGaN} \mathrm{x}=0.068$ sample which was grown at $780^{\circ} \mathrm{C}$. The reactor pressure was held constant at 200 Torr. The $\mathrm{NH}_{3}$ and $\mathrm{N}_{2}$ flows were set at $6 \mathrm{l} / \mathrm{min}$ each. An additional flow of $\mathrm{H}_{2}\left(\sim 400 \mathrm{~cm}^{3} / \mathrm{min}\right)$ was employed as a carrier gas.

Trimethylgallium, triethylaluminum, and trimethylindium are employed as metalorganic precursors. The samples were doped $n$-type with silane up to a concentration of $n=$ $2 \times 10^{19} \mathrm{~cm}^{-3}$. While the exact level of Si-doping is expected to affect the quantitative optical properties, SIMS data has verified that the Si level is very reproducible for all of the samples studied. The indium composition was determined by both Rutherford Backscattering Spectroscopy (RBS), configured to minimize channeling effects, as well as $\mathrm{x}$-ray diffraction (XRD) experiments. Analysis of the x-ray diffraction assumed that the films were fully pseudomorphic, which was supported by the strong thickness fringes seen in the data. In general, the In composition measured with the two techniques agreed within $0.5 \%$. The In compositions determined by the two techniques are summarized in Table 1.

\begin{tabular}{|c|c|c|}
\hline Sample & \% In by RBS & \% In by XRD \\
\hline 1 & 0 & 0 \\
\hline 2 & 1.09 & 0.75 \\
\hline 3 & 3.94 & 3.71 \\
\hline 4 & 6.82 & 6.31 \\
\hline
\end{tabular}

TABLE 1: Indium composition of InGaN bulk epilayers determined by RBS and XRD measurements. The samples will be referred to by the RBS values.

Time-integrated photoluminescence measurements were performed using a $\mathrm{HeCd}$ laser $(325 \mathrm{~nm})$ at a low power density of approximately $30 \mathrm{~W} / \mathrm{cm}^{2}$. A 0.3 meter spectrometer with an integrated UV enhanced CCD detector was used, with a spectral resolution of approximately $0.2 \mathrm{~nm}$. Time-resolved photoluminescence measurements were performed using a frequency tripled Ti: sapphire laser with a 1 psec pulsewidth, 82 $\mathrm{MHz}$ repetition rate and wavelength of $260 \mathrm{~nm}$. Average power densities of $10 \mathrm{~W} / \mathrm{cm}^{2}$ were typically used. The PL was collected into a 0.5 meter spectrometer and a 
Hamamatsu model 4334 streakscope with resolutions of $0.5 \mathrm{~nm}$ and $15 \mathrm{psec}$, respectively. For all measurements, the samples were mounted in a closed cycle cryostat to enable measurements over the $4 \mathrm{~K}-300 \mathrm{~K}$ temperature range.

\section{TIME-INTEGRATED PHOTOLUMINESCENCE RESULTS}

In Figure 1a, we show room temperature photoluminescence data of the various InGaN epilayers. A clear increase in the integrated PL intensity is seen as In composition is increased, and the wavelength shifts from 363-397 nm. The temperature dependent integrated PL intensity of the InGaN epilayers is shown in Figure 1b. An interesting feature of the data is that for $\mathrm{GaN}$ and the $\mathrm{InGaN}$ ( $\mathrm{x}=0.011$ ) sample, a very quick drop in PL intensity is seen with increasing temperature. In contrast, the samples with higher In composition don't exhibit a significant drop in intensity until approx. 50$100 \mathrm{~K}$. This behavior is suggestive that carrier localization may be operative in the $\mathrm{x} \geq$ 0.039 samples at low temperatures.
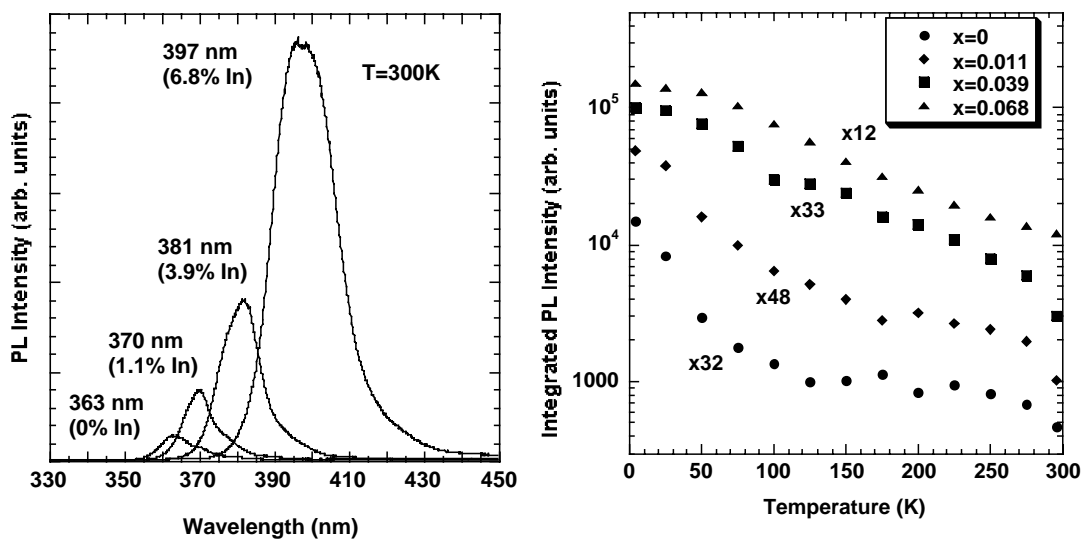

Figure 1: (a) Room temperature time-integrated PL of various InGaN epilayers (b) temperature dependence of integrated PL intensities. The data are labeled with the total drop in integrated PL intensity from $4 \mathrm{~K}-300 \mathrm{~K}$ for each sample.

We have replotted the temperature dependent PL data on an Arrhenius plot in Figure 2. The data is fit with the following formula

$$
\mathrm{I}=\mathrm{I}_{0} /\left[1+\alpha \exp \left(-\mathrm{E}_{\mathrm{a}} / \mathrm{kT}\right)\right] .
$$

Here the non-radiative decay is assumed to be thermally activated such that the nonradiative lifetime $\tau_{n r}=\tau_{o} \exp \left(E_{a} / k T\right)$ and $E_{a}$ is the activation energy for PL quenching. The parameter $\alpha$ is equal to $\tau_{\mathrm{r}} / \tau_{\mathrm{o}}$ where $\tau_{\mathrm{r}}$ is the radiative lifetime. From the $\mathrm{E}_{\mathrm{a}}$ values, we see a systematic increase in the activation energy as the indium composition is increased. Leroux et. al. [12] performed a similar analysis of $\mathrm{GaN}$ epilayers and found that the activation energy was approximately $25 \mathrm{meV}$, which is well correlated with the exciton binding energy. They suggested that the dissociation of the exciton into its charged electron and hole components results in more effective trapping of carriers at charged 
non-radiative recombination sites (e.g. dislocations). In contrast, our $\mathrm{GaN}$ epilayer shows a much lower activation energy. The discrepancy in our results may be explained by the fact that our samples have a relatively high n-type doping level that exceeds the Mott density required to effectively screen excitons [13], and our data is expected to reflect the effectiveness of non-radiative recombination on free carriers throughout the temperature range. As a comparison between our results and other reports on InGaN materials, we note that a large variation in $\mathrm{E}_{\mathrm{a}}$ values for $\mathrm{InGaN}$ epilayers and MQWs has been reported in the literature. Smith et. al. [14] report $\mathrm{E}_{\mathrm{a}}=56 \mathrm{meV}$ for $\mathrm{a} x=0.12$ epilayer for which the authors predict exciton recombination is dominant. Teo et al. [15] report on $\operatorname{In}_{0.20} \mathrm{Ga}_{0.80} \mathrm{~N}$ MQWs with $E_{a}$ values of $63 \mathrm{meV}$. Our results indicate significantly lower activation energies for free carrier recombination in bulk samples with lower In composition. Furthermore, in our samples, the addition of indium clearly serves to reduce the effect of non-radiative recombination, and thereby a higher optical efficiency over a larger temperature range is maintained. The high carrier density and free carrier regime is relevant for the operating conditions of InGaN laser diodes.

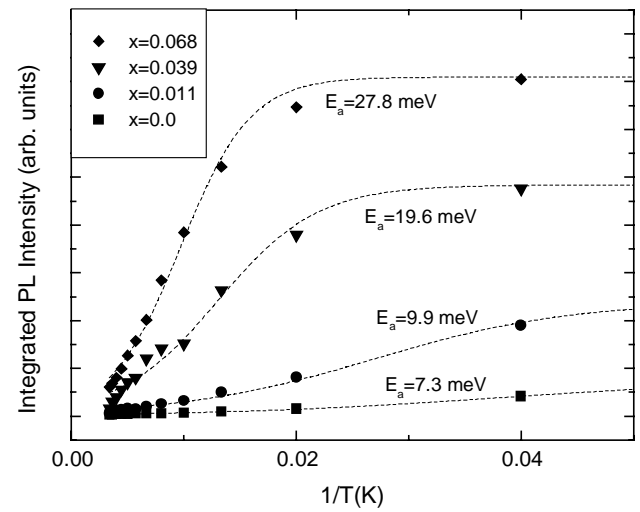

Figure 2: Temperature dependent PL intensity data fit with equation 1.

\section{TIME-RESOLVED PHOTOLUMINESCENCE RESULTS}

Further insight into the possible role of localization may be obtained through examination of the time decay of the PL intensity. The decay data can be fit with a single exponential function. At low temperature, we see a clear increase in the measured PL decay time as In composition is increased. To explore this further, we have spectrally resolved the $\mathrm{T}=4 \mathrm{~K}$ data to determine if there is a spectral dependence of the decay time (not shown). None of the samples showed an appreciable variation ( $>30 \mathrm{psec}$ ) in decay time except for the sample with the highest In composition $(\mathrm{x}=0.068)$. For this sample, we see a variation of approximately $150 \mathrm{psec}(450-600 \mathrm{psec})$ across the resonance at $4 \mathrm{~K}$. A clear spectral variation in the decay time persists until approximately $220 \mathrm{~K}$, suggesting that the carriers do not fully come into equilibrium until this relatively high temperature for this sample.

A full temperature dependence of the decay times was measured for each sample, and this data is shown in Figure 3. The decay times were measured at the spectral position of peak PL intensity at all temperatures. It is expected that an increase 
in the decay time with temperature would be seen if the samples were dominated by radiative recombination [16]. The fact that the $\mathrm{GaN}$ and the $\mathrm{InGaN}(\mathrm{x}=0.011)$ samples show a strong decrease in the decay time at low temperatures suggests that non-radiative mechanisms dominate over the entire temperature range. Furthermore, the strong similarity between these two samples suggests that $1 \%$ of In does not significantly affect the recombination processes. The samples with higher In composition, however, show constant or slightly increasing decay times up to approximately $25-50 \mathrm{~K}$ followed by a decrease in decay time. The distinct behavior of these samples in the low temperature regime is consistent with the carriers being localized at low temperatures with increasing thermal delocalization at temperatures in the 50-100K range. Our results for the $\mathrm{x}=0.068$ sample, in particular the dominance of non-radiative recombination for $\mathrm{T}>100 \mathrm{~K}$ and the relatively small variation of decay times over the entire temperature range, are similar to the temperature dependence seen in $\operatorname{In}_{0.10} \mathrm{Ga}_{0.90} \mathrm{~N}$ MQW structures [17]. Overall, our data demonstrate a clear increase of the room temperature non-radiative decay times as In composition is increased, which is well correlated with the increased localization seen in the higher In samples.

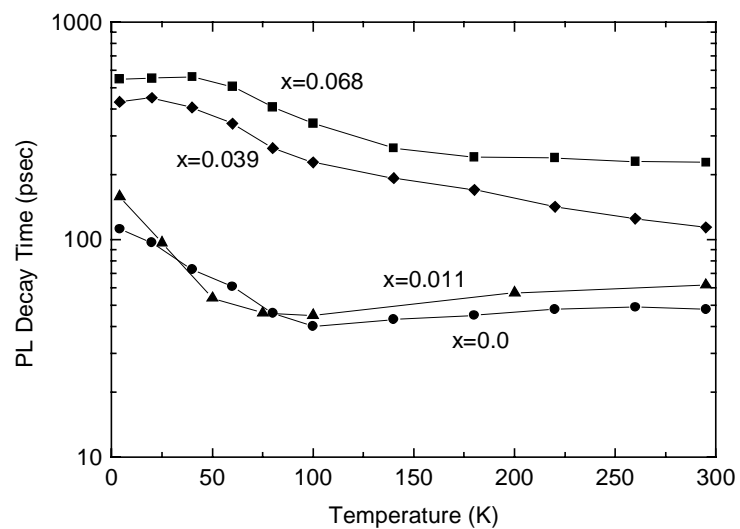

Figure 3: PL decay times as a function of temperature for GaN and $\mathrm{InGaN}$ epilayers.

\section{CONCLUSIONS}

We have conducted a spectroscopic investigation of InGaN epilayers in an effort to determine how the presence of In affects the optical properties. The strong increase in time-integrated PL intensity that is seen with increasing In is well correlated with increasing thermal activation energies for PL quenching. These activation energies range from 7-28 meV. The temperature dependence of the PL decay times suggests that the samples with higher In $(x \geq 0.039)$ show carrier localization effects at low temperatures; these effects are most clearly seen in the spectral dependence of PL decay times for the $x=0.068$ sample. Overall, the results indicate that carrier localization is enhanced with just the addition of a few percent of In and radiative recombination may dominate in the samples with higher In $(x \geq 0.039)$ at lower temperatures. However, nonradiative recombination dominates for much of the temperature range and the fact that the room temperature PL decay times increase with increasing In demonstrates that nonradiative recombination is less effective with increasing In. Since the localization effects

F99W11.41 
in these low In composition samples are relatively weak, it is not clear from our data that carrier localization alone determines the longer non-radiative decay times at room temperature. A modification of the density, capture efficiency or activation of nonradiative centers due to the presence of In would also be consistent with the data.

\section{ACKNOWLEDGEMENTS}

The authors gratefully acknowledge technical discussions with Weng Chow, Ian Fritz and Nancy Missert. Sandia is a multiprogram laboratory operated by Sandia Corporation, a Lockheed Martin company, for the US Dept. of Energy under contract DE-AC04-94AL85000.

\section{REFERENCES}

1. E. S. Jeon, V. Kozlov, Y.-K. Song, A. Vertikov, M. Kuball, A. V. Nurmikko, H. Liu, C. Chen, R. S. Kern, C. P. Kuo and M. G. Craford, Appl. Phys. Lett., 69, 4194 (1996).

2. S. Chichibu, T. Azuhata, T. Sota and S. Nakamura, Appl. Phys. Lett., 70, 2822 (1997).

3. T. Wang, D. Nakagawa, M. Lachab, T. Sugahara, S. Sakai, Appl. Phys. Lett., 74, 3128 (1999) and references therein.

4. S.F.Chichibu, A.Shikanai, T.Deguchi, A.Setoguchi, R.Nakai, H.Nakanishi, K.Wada, S.P.DenBaars, T.Sota, and S.Nakamura, Jpn. J. Appl. Phys. 39, Part 1 (2000) (to be published)

5. Y. Narukawa, S. Saijou, Y. Kawakami, S. Fugita, t. Mukai, S. Nakamura, Appl. Phys. Lett., 74, 558 (1999).

6. H. Kumano, K. Hoshi, S. Tanaka, I. Suemune, X. Q. Shen, P. Riblet, P. Ramvall and Y. Aoyagi, Appl. Phys. Lett., 75, 2879 (1999).

7. S. Chichibu, H. Marchand, M. S. Minsky, S. Keller, P. T. Fini, J. P. Ibbetson, S. B. Fleischer, J. S. Speck, J. E. Bowers, E. Hu, U. K. Mishra, S. P. Denbaars, T. Deguchi, T. Sota, S. Nakamura, Appl. Phys. Lett., 74, 1460 (1999).

8. T. Sugahara, M. Hao, T. Wang, D. Nakagawa, Y. Naoi, K. Nishino, S. Sakai, Jpn. J. Appl. Phys., 37, L1195 (1998).

9. S. Keller, U. Mishra, S. Denbaars, W. Siefert, Jpn. J. Appl. Phys., 37, L431 (1998).

10. P. Riblet, H. Hirayama, A. Kinoshita, A. Hirata, T. Sugano, Y. Aoyagi, Appl. Phys. Lett., 75, 2241 (1999).

11. V. Fiorentini, F. Bernadini, Phys. Rev. B, 60, 8849 (1999).

12. M. Leroux, N. Grandjean, B. Beaumont, G. Nataf, F. Semond, J. Massies, P. Gibart, J. Appl. Phys., 86, 3721 (1999).

13. W. W. Chow, A. Knorr, S. W. Koch, Appl. Phys. Lett., 67, 754 (1995).

14. M. Smith, G. D. Chen, J. Y. Lin, H. X. Jiang, M. Asif Khan, Q. Chen, Appl. Phys. Lett., 69, 2837 (1996).

15. K. L. Teo, J. S. Colton, P. Y. Yu, E. R. Weber, M. F. Li, W. Liu, K. Uchida, H. Tokunaga, N. Akutsu, K. Matsumoto, Appl. Phys. Lett., 73, 1697 (1998).

16. B. Barry Webb and E. W. Williams in Semiconductors and Semimetals vol. 8 (R. K. Williardson and A. C. Beer, editors, Academic Press, New York, 1972) chapter 4.

17. Y. Narukawa, Y. Kawakami, S. Fugita, S. Nakamura, Phys. Rev. B, 59, 10283 (1999). 
F99W11.41

https://doi.org/10.1557/S1092578300004981 Published online by Cambridge University Press 